\title{
Theory of muscle contraction mechanism with cooperative interaction among crossbridges
}

\author{
Toshio Mitsui ${ }^{1}$ and Hiroyuki Ohshima ${ }^{2}$ \\ ${ }^{1}$ Nakasuji-yamate, 3-6-24, Takarazuka, Hyogo 665-0875, Japan \\ ${ }^{2}$ Faculty of Pharmaceutical Sciences, Tokyo University of Science, 2641, Yamazaki, Noda, Chiba 278-8510, Japan
}

Received November 10, 2011; accepted December 27, 2011

\begin{abstract}
The power stroke model was criticized and a model was proposed for muscle contraction mechanism (Mitsui, 1999). The proposed model was further developed and calculations based on the model well reproduced major experimental data on the steady filament sliding (Mitsui and Ohshima, 2008) and on the transient phenomena (Mitsui, Takai and Ohshima, 2011). In this review more weight is put on explanation of the basic ideas of the model, especially logical necessity of the model, leaving mathematical details to the above-mentioned papers. A thermodynamic relationship that any models based upon the sliding filament theory should fulfill is derived. The model which fulfills the thermodynamic relationship is constructed on the assumption that a myosin head bound to an actin filament forms a complex with three actin molecules. In shortening muscles, the complex moves along the actin filament changing the partner actin molecules with steps of about $5.5 \mathrm{~nm}$. This process is made possible through cooperative interaction among crossbridges. The ATP hydrolysis energy is liberated by fraction at each step through chemical reactions between myosin and actin molecules. The cooperativity among crossbridges disappears in length-clamped muscles, in agreement with experimental observations that the crossbridge produces force independently in the isometric tetanus state. The distance of the head movement per ATP hydrolysis cycle is expected to be about $5.5 \mathrm{~nm}$ or a few times of it under the condition of the in vitro single head experiments. Calculation results are surveyed illustrating that they are in good agreement with major experimental observations.
\end{abstract}

Corresponding author: Toshio Mitsui, Nakasuji-yamate, 3-6-24, Takarazuka, Hyogo 665-0875, Japan.

e-mail: t-mitsui@jttk.zaq.ne.jp
Key words: muscle stffness, force-velocity relation, energy liberation rate, isometric tension transient, isotonic velocity transient

\section{Introduction}

The power stroke model was criticized and a model was proposed for the muscle contraction mechanism in ref. 1 . The model was further developed in refs. 2, 3. Calculations by the model well explained major experimental data on the steady filament sliding ${ }^{2}$ and on the transient phenomena ${ }^{3}$ (as surveyed later in Sect. 5). Some readers of refs. 2, 3, however, commented that it was difficult to understand the model since the explanations were too brief on some basic ideas, especially on logical necessity of the model. In this review, we will try to fill up the deficiency.

A thermodynamic relationship which any model based upon the sliding filament theory should fulfill is derived in Sect. 2. Requirements asked to any models are listed in Sect. 3. In Sect. 4, basic concepts of the proposed model are explained. The requirements are fulfilled by considering cooperative interaction among crossbridges and by fractional liberation of ATP energy through chemical reactions between myosin and actin molecules. In Sect. 5, calculation results by the model are surveyed. In Sect. 6, summary and discussion are given.

\section{Derivation of a thermodynamic relationship}

Generally the first step to construct a molecular model in material physics is to look for a thermodynamic relationship among parameters to appear in models and put restrictions 
on the construction of models. Let us follow this procedure for a study of muscle contraction.

\subsection{Work done by one crossbridge using ATP hydrolysis energy}

It is assumed that the myosin head exerts force on actin filament only when it is attached to actin. The mean force exerted on the actin filament by one attached myosin head is denoted as $p$ and the translation distance of the attached head during one ATP hydrolysis cycle as $D$. Then $p D$ gives the work done by the myosin head utilizing the ATP hydrolysis energy, $e_{\mathrm{A}}$. Measured macroscopic quantities during muscle contraction are the tension $T$, contraction velocity $V$ and the rate of heat production $H$. Usually the contraction velocity $V$ is defined by the shortening velocity of muscle of length $L_{\mathrm{ml}}$ divided by $L_{\mathrm{ml}}$ so that unit of $V$ is $\mathrm{s}^{-1}$. The rate of muscle work $W$ is $T V$ and the rate of energy production is $W+H$. The portion of $e_{\mathrm{A}}$ used for work is given by $e_{\mathrm{A}} W /(W+H)$, and therefore, by the first law of thermodynamics, we have

$$
p D=e_{\mathrm{A}} W /(W+H) \text {. }
$$

\subsection{Relation between $p$ and $T$ in the sliding filament theory}

Figure 1 schematically shows a portion of the sarcomere producing the tension $T$ at full filament overlap: (a) side view and (b) projection of the sarcomere along the filament axis. The projection consists of rhombic cells of edge $a$ or of hexagons. The hexagon contains one myosin filament and has the same area as the rhombic cell.

Since the tension $T$ is given in units of $\mathrm{N} / \mathrm{m}^{2}$, let us consider a flat cylinder whose bases are $1 \mathrm{~m}^{2}$ wide and vertical to the filaments and thickness is the half sarcomere length $s / 2$ between the planes $Z$ and $M$. Such cylinders are indicated as $\mathrm{C} 1$ and $\mathrm{C} 2$ in Figure 1(a). The $\mathrm{Z}$ plane is considered fixed and thus the tension $T$ is downward. Then the actin filaments in $\mathrm{C} 1$ exert upward force on myosin filaments balancing the tension $T$, while the myosin filaments in $\mathrm{C} 2$ exert upward force on actin filaments balancing $T$. In such ways, each flat cylinder produces the tension $T$ in the sliding filament theory. Below we shall consider $\mathrm{C} 1$.

The number of myosin heads in $\mathrm{C} 1$ is denoted as $N_{\mathrm{hs}}$, whose numerical value can be obtained as follows. The edge of the rhombus unit cell is indicated as $a$ in Figure 1(b). It is known that $a$ varies depending upon $T$ but, as an approximation, $a$ may be set equal to $42 \mathrm{~nm}$ following ref. 4 . Then the area of the unit cell is $a^{2} \sin 60^{\circ}=1528 \times 10^{-18} \mathrm{~m}^{2}$. Microscopic photographs of muscle suggest that the ratio of sarcomere area in the base of $\mathrm{C} 1$ is not very far from 1 , and is set as 0.9 as an approximation following ref. 4 . Then the number of myosin filaments in $\mathrm{C} 1$ is $0.9 /\left(a^{2} \sin 60^{\circ}\right)=$ $5.89 \times 10^{14} \mathrm{~m}^{-2}$. The period of the myosin filament is denoted as $d$, and set $d$ equal to $14.3 \mathrm{~nm}$ as in ref. 4 . The myosin filament has a three-fold rotation symmetry and has 6 myosin (a)

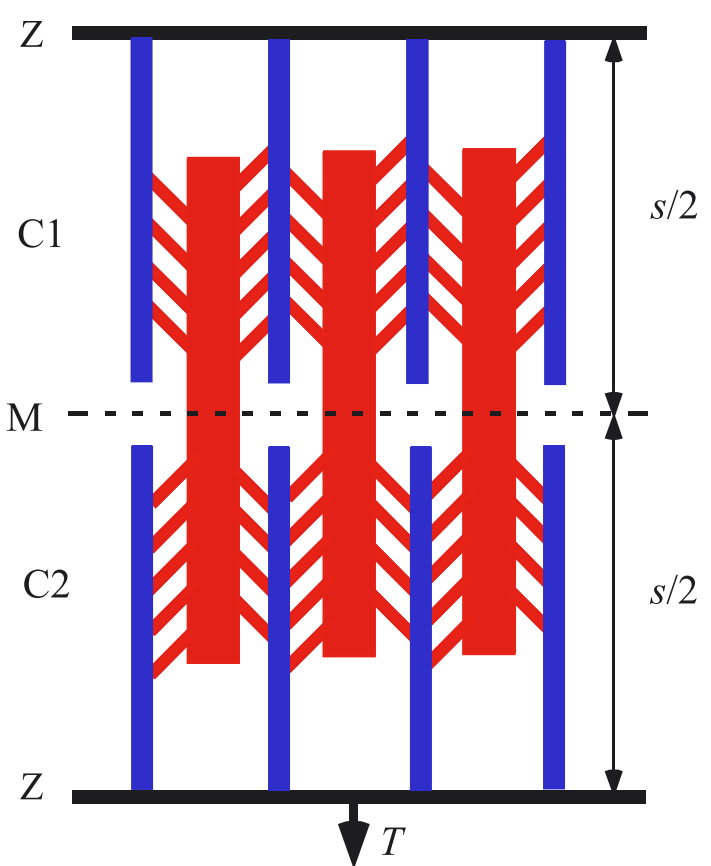

(b)

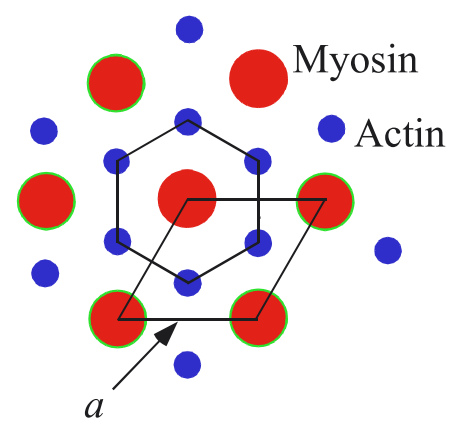

Figure 1 A portion of the sarcomere at the full filament overlap. (a) Side view. Myosin filaments are shown in red and the actin filament in blue. (The actually number of myosin molecules per half myosin filament is about 50.) The thickness of half sarcomere is $s / 2$. The upper $\mathrm{Z}$ membrane is fixed and the tension $T$ is downward. See text for $\mathrm{C} 1$ and $\mathrm{C} 2$. (b) Projection of the sarcomere along the filament axis. The projection consists of rhombic cells of edge $a$ or of hexagons. The hexagon contains one myosin filament and has the same area as the rhombic cell.

heads per $d$. The length of myosin filament minus that of the bare zone is about $1.43 \mu \mathrm{m}$. Thus the number of myosin heads on one myosin filament in half sarcomere is $6 \times\left(1.43 \times 10^{-6} / 2\right) / 14.3 \times 10^{-9}=300$. Thus we have an approximate value of $N_{\text {hs }}$ as

$$
N_{\text {hs }}=300 \times 5.89 \times 10^{14} \mathrm{~m}^{-2}=1.76 \times 10^{17} \mathrm{~m}^{-2}
$$

The symbol $r$ is defined as the ratio of the number of myosin heads attached to an actin filament per total number of myosin heads at the same moment. Then the relation between the mean force $p$ produced by one attached head and the tension $T$ is given by 


$$
p=T /\left(r N_{\mathrm{hs}}\right)
$$

\subsection{Thermodynamic relationship}

Combining Eqs. 1 and 3 with $W=T V$ gives

$$
D / r=e_{\mathrm{A}} N_{\mathrm{hs}} V /(W+H)
$$

This is the required thermodynamic relationship which any model based on the sliding filament theory should satisfy. If the units of the quantities in Eq. 4 are presented in parentheses, they are $D(\mathrm{~m}), e_{\mathrm{A}}(\mathrm{J}), N_{\mathrm{hs}}\left(1 / \mathrm{m}^{2}\right), V(1 / \mathrm{s}),(W+H)$ $\left(\mathrm{J} / \mathrm{m}^{3} \mathrm{~s}\right)$, so that the unit of the right-handed term is $(\mathrm{J})\left(1 / \mathrm{m}^{2}\right)$ $(1 / \mathrm{s})\left(\mathrm{m}^{3} \mathrm{~s}\right) / \mathrm{J}=\mathrm{m}$, the same as the unit of $D / r$.

As $T$ approaches to $0, W=T V$ becomes negligible compared to $H$, and Eq. 4 becomes

$$
D / r=e_{\mathrm{A}} N_{\mathrm{hs}} V / H
$$

Let us use $e_{\mathrm{A}}=8.0 \times 10^{-20} \mathrm{~J} /$ molecule, $V_{\max }=2.25(1 / \mathrm{s})(V$ under no load) $)^{5}, H=35.2 \mathrm{~kW} / \mathrm{m}^{2}$ at $T=0$ at $0^{\circ} \mathrm{C}^{6}$, as was done in Sect. 2.1 of ref. 2. Then Eq. 5 gives

$$
D / r=900 \mathrm{~nm} \text {, for } T=0 \text { at } 0^{\circ} \mathrm{C}
$$

This value of $D / r$ is large but those of a similar order of magnitude are obtained from other experimental data in the next section.

\section{4 $\mathrm{D} / \mathrm{r}$ values estimated from the data by Harada et al. $^{7}$}

Harada et al. ${ }^{7}$ observed the motion of actin filaments on a glass strip covered by myosin molecules, and measured the velocity of the actin filament and rate of hydrolysis of ATP. They estimated from their data that $D$ is larger than $60 \mathrm{~nm}$ at $30^{\circ} \mathrm{C}$ and than $200 \mathrm{~nm}$ at $20^{\circ} \mathrm{C}$. As mentioned by several authors, however, their way to estimate $D$ seems too speculative. Nevertheless, the order of magnitude of $D / r$ can be estimated from their data as follows.

According to Harada et al. ${ }^{7}$, the mean sliding velocity of the actin filaments does not depend upon the filament length. They determined the rate of hydrolysis of ATP by the Pi production rate per $1 \mathrm{~nm}$ of the actin filament. Here let us simplify their system by supposing the following model. Myosin molecules are arranged on a straight line with the mutual separation of $d_{\mathrm{H}}$ on the glass strip. The length of the line is more than $1 \mathrm{~m}$. A straight actin filament of $1 \mathrm{~m}$ is on the myosin line and moves along it. Now the stress $T$ is regarded as a friction force against the actin filament movement. Then relations similar to Eqs. 1, 3 and 4 can be derived. If the quantities concerning the experiment by Harada et al. ${ }^{7}$ are expressed with suffixes $H$, Eq. 4 becomes

$$
D_{\mathrm{H}} / r_{\mathrm{H}}=e_{\mathrm{A}} N_{\mathrm{H}} V_{\mathrm{H}} /\left(H_{\mathrm{H}}+W_{\mathrm{H}}\right)
$$

Harada et al. determined the average distance between myosin molecules on the glass strip by electron microscope as about $12 \mathrm{~nm}$. Hence we set.

$$
d_{\mathrm{H}}=12 \mathrm{~nm}
$$

Since the length of the actin filament is $1 \mathrm{~m}$ and myosin molecule is two-headed, we have

$$
N_{\mathrm{H}}=2 / d_{\mathrm{H}}=2 / 12 \times 10^{-9}=0.17 \times 10^{9}
$$

In Table 1 of the paper by Harada et al. ${ }^{7}$, values of $v(\mu \mathrm{m} / \mathrm{s})$ and $(\mathrm{Pi} / \mathrm{s}$ per $\mathrm{nm})$ are given. With our notations, $V_{\mathrm{H}}(\mathrm{m} / \mathrm{s})=$ $v \times 10^{-6}$ and $H_{\mathrm{H}}+W_{\mathrm{H}}(\mathrm{J} / \mathrm{s}$ per $\mathrm{m})=\varepsilon_{\mathrm{ATP}}(\mathrm{Pi} / \mathrm{s}$ per $\mathrm{nm}) \times 10^{9}$. Then Eq. 7 becomes

$$
D_{\mathrm{H}}(\mathrm{nm}) / r_{\mathrm{H}}=170 v /(\mathrm{Pi} / \mathrm{s} \text { per nm })
$$

By using the values given in Table 1 of the paper by Harada et al. ${ }^{7}$ and $N_{\mathrm{H}}$ in Eq. 9, we have

$$
\begin{aligned}
& D_{\mathrm{H}} / r_{\mathrm{H}}=850 \mathrm{~nm} \text { for actin filament at } 22^{\circ} \mathrm{C} \\
& D_{\mathrm{H}} / r_{\mathrm{H}}=350 \mathrm{~nm} \text { for actin filament at } 30^{\circ} \mathrm{C} \\
& D_{\mathrm{H}} / r_{\mathrm{H}}=1280 \mathrm{~nm} \text { For thin filament at } 22^{\circ} \mathrm{C}
\end{aligned}
$$

These values of $D_{\mathrm{H}} / r_{\mathrm{H}}$ are as large as $D / r=900 \mathrm{~nm}$ in Eq. 6 .

Presumably, one can get valuable information on the mechanism of myosin sliding on the actin filament by extending the ingenious experiment by Harada et $\mathrm{ll}^{7}$, changing experimental parameters and using muscles of different kinds.

\section{Requirements for any models based upon the sliding filament theory}

Requirements which any models based upon the sliding filament theory should fulfill may be listed as follows.

(1) Large $D / r$ in shortening muscle

According to Eq. $6, D / r=900 \mathrm{~nm}$ for $T=0$ at $0^{\circ} \mathrm{C}$. This relation is derived solely based upon the filament sliding theory and thus any model based upon the sliding filament theory should give this order of magnitude of $D / r$.

(2) Chemical reactions associated with the large $D / r$

Coupling between the force production and chemical reaction should be explained in a reasonable manner.

(3) Crossbridges produces force independently in the isometric tetanus state

Ramsey and Street ${ }^{8}$ and Gordon et al. ${ }^{9}$ examined variation of active tension with overlap between myosin and actin filaments in fibers of frog muscles. They observed a roughly linear decline of active tension with extension of the fiber beyond the length at which it gave maximum force (cf. Fig. 1 in ref. 10). These observations indicate that crossbridges produces force independently when the muscle length is clamped.

(4) Small $D$ observed in experiments with single myosin molecule in vitro

Molloy et al. ${ }^{11}$ studied the movement and force generation produced by a single myosin head and found the movement during one ATP hydrolysis cycle to be about $4 \mathrm{~nm}$. Kitamura et al. ${ }^{12}$ developed a new assay for direct manipulation of S-1 making visualization possible with a fluorescent label. Their results show that a myosin head moves along the actin filament with $5.3 \mathrm{~nm}$ steps, often producing displacement of 11 to $30 \mathrm{~nm}$ for each ATP hydrolysis cycle. 
(5) The model should explain major experimental data on muscle properties or behaviors.

\section{Construction of a new model}

\subsection{Ratio $r$ of the myosin heads attached to actin filaments to all heads}

In Sect. 2.2, the ratio of the number of myosin heads attached to actin filament per total number is denoted as $r$. Here let us estimate the value of $r$. The isometric tension per head is denoted as $p_{0}$. Ishijima et al. ${ }^{13}$ did single-molecule analysis of the actomyosin motor using nano-manipulation. Their experimental results indicate that $p_{0}$ is close to $5.7 \mathrm{pN}$. We adopt this value:

$$
p_{0}=5.7 \mathrm{pN} \text {. }
$$

According to Eq. 3, $p=T /\left(r N_{\mathrm{hs}}\right)$, and thus denoting the macroscopic isometric tension as $T_{0}$, we have $r=T_{0} /\left(p_{0} N_{\mathrm{hs}}\right)$. If we use the above value of $p_{0}$ and $N_{\mathrm{hs}}$ in Eq. 2 with the experimental values of $T_{0}=4.1 \times 10^{5} \mathrm{~N} / \mathrm{m}^{2}$ in ref. 5 , we have

$$
r=0.41 \text {, at } T=T_{0} .
$$

$\mathrm{X}$-ray data are favorable to this $r$ value. By X-ray diffraction study of the equatorial reflections from a sartorius muscle, Matsubara et al. ${ }^{14}$ estimated that about $80 \%$ of myosin heads migrate around the thin filament in isometric tetanus muscle. This percentage is higher (about 92 99\%) according to Yagi et al $^{15}$. Since these percentages are about twice $r=41 \%$, it seems reasonable to suppose that one of two heads of a myosin molecule attaches to actin and produces positive or negative force while the other exists in the vicinity of the thin filament as non-attached relief.

Podolsky et al. ${ }^{16}$, Huxley ${ }^{17}$, Huxley and Kress ${ }^{18}$ and Yagi et al. ${ }^{15}$ observed that the intensity ratio of the $[1,0]$ and $[1,1]$ equatorial reflections increases only minimally as shortening velocity increases, indicating that the total number of myosin heads in the vicinity of the actin filament decreases only slightly. In the power stroke model, the number of the myosin heads producing tension depends upon the tension and the X-ray observation is explained by assuming that weakly attached crossbridges exist in addition to strongly attached crossbridges and the muscle stiffness is determined by the strongly attached crossbridges. Then a question arises how this weakly attached crossbridge is related to the force production mechanism. It seems reasonable to consider that both the strongly attached and weakly attached crossbridges are substantially related to the force production in positive or negative ways. In our model both are counted to calculate the ratio $r$, i.e., it is assumed that, as an approximation, $r$ is constant and independent of the shortening velocity, as the X-ray data suggest. Referring to Eq. 13, the following value of $r$ seems close to reality.

$$
r=0.41, \text { for any } T
$$

\subsection{Formation of complex $\mathrm{MA}_{3}$ of myosin head and actin molecules}

Andreeva et $a l .{ }^{19}$ and Xiao et al. ${ }^{20}$ studied the way of binding of a myosin head to an actin filament by using tryptic digestion of myofibrils and measuring optical polarization and dichroism. They concluded that in the rigor rabbit psoas muscle each myosin head binds to two actin monomers in a thin filament ${ }^{20}$, suggesting the possibility that the myosin head may first bind to one and then to two monomers in the actin filament ${ }^{19}$.

Figure 2 shows an example of possible mechanism of how such binding change occurs. In Figure 2(a), an ATPactivated single myosin head (S-1) is indicated as $\mathrm{M}$, which sits at a position apart from the actin filament. The actin filament is represented by the periodic array of potential wells which correspond to the possible binding sites for $\mathrm{M}$. The wells are arranged with a period of the strand $L$ :

$$
L=5.46 \mathrm{~nm}
$$

The helical structure of the actin filament will disturb the periodic potential arrangement but its effect does not seem essential and is neglected here. Figure 2(b) shows the state at the moment $\mathrm{M}$ attaches to actin 1. Attachment of the myosin head may cause large strain in actin molecules. Protein molecules are structurally polar. (Note that G-actin has the permanent dipole moment of 600 Debye $^{21}$.) Hence the actin filament is piezoelectric. Consequently, it is a possibility that the strain produces an electric polarization as symbolically indicated by the electric charges - in actin 1 and + in actin 2 in Figure 2(c). The myosin head is negatively charged and the Coulomb interaction between the head charge and the induced polarization charges raise the potential well at actin molecule 1 and lower the well at actin 2 , resulting in the potential energy distribution for the head shown in Figure 1(c), which might be wide over the two actin molecules and has two narrow inner potential wells at the actin molecule 1 and 2, such that $\mathrm{M}$ can jump from one to the other due to the thermal fluctuation and is statistically distributed in these wells as schematically drawn in Figure 2(d). The negative charge of the head will tend to stabilize the charges caused by the polarization in wells 1 and 2 . The head at well 2 will newly produce the polarization charge in well 2 and + in well 3 similarly to the case of (c) but somewhat differently due to the difference in prehistory from (c). The induced charges in this case are symbolically shown by small - and + in (d). Accordingly, the structural changes in (d) will be similar to (c) but somewhat different from (c). The potential distribution is supposed to be such as shown in (d). Presumably the myosin head bound to Actin 1 and 2 is observed in the experiment of ref. 19 and ref. 20 .

Figure 2(d) shows the potential barrier $U^{*}$ which a myosin head $M$ has to cross over to move from well 2 to 3 . In our model, it is assumed that $U^{*}$ is relatively large if there is no force acting on the myosin head but sensitively depends upon the force on the head, as discussed in detail in Sect. 
(a)

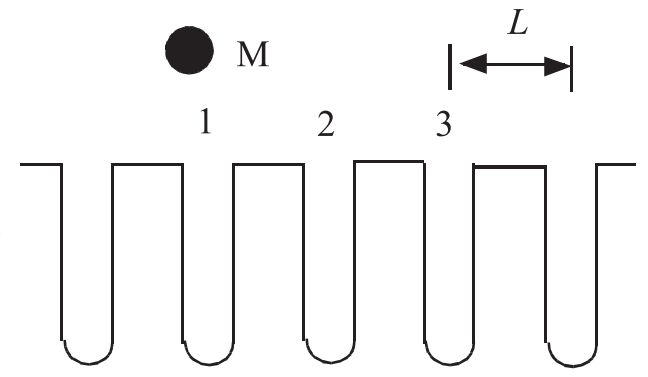

(b)

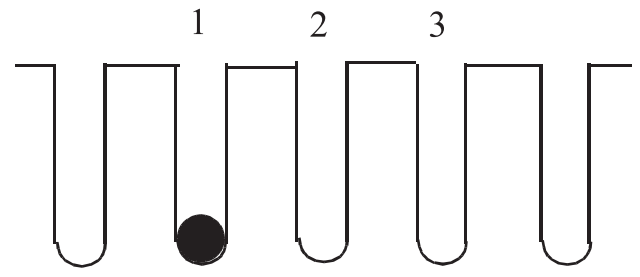

(c)

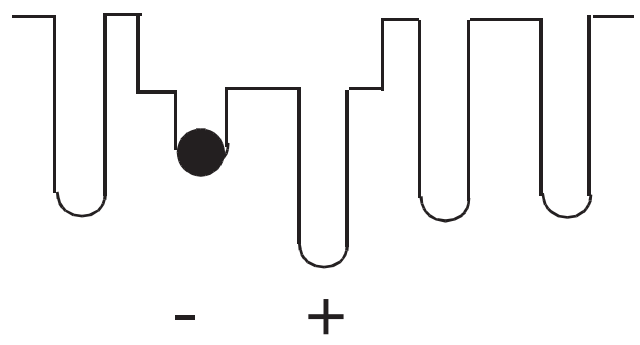

$12=3$

(d)

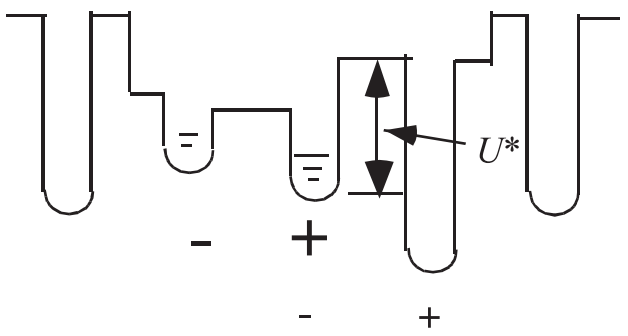

Figure 2 Diagram showing sequential changes in the potential of force acting on ATP-activated single myosin head M (subfragment-1) when $\mathrm{M}$ binds to an actin filament. The numbers 1,2 and 3 are assigned to the potential wells at the binding sites on actin molecules on the same strand. (a) Periodic potential distribution when $M$ is sitting at a position apart from the actin filament. (b) Just after $M$ attaches to actin 1. (c) Molecules deforms and potential distribution changes. (d) Equilibrium potential distribution. Generally $\mathrm{M}$ is statistically distributed in wells 1 and 2.

4.3. Accordingly, the head M moves by about $5.46 \mathrm{~nm}$ from well 1 to 2 and stops if there is no force on the head. As mentioned in Sect. 3, Kitamura et al. ${ }^{12}$ observed that a single myosin head moves by $5.3 \mathrm{~nm}$ or about a few times of $5.3 \mathrm{~nm}$ along the actin filament during one ATP hydrolysis cycle in vitro. This observation seem to indicate that $U^{*}$ is (a)

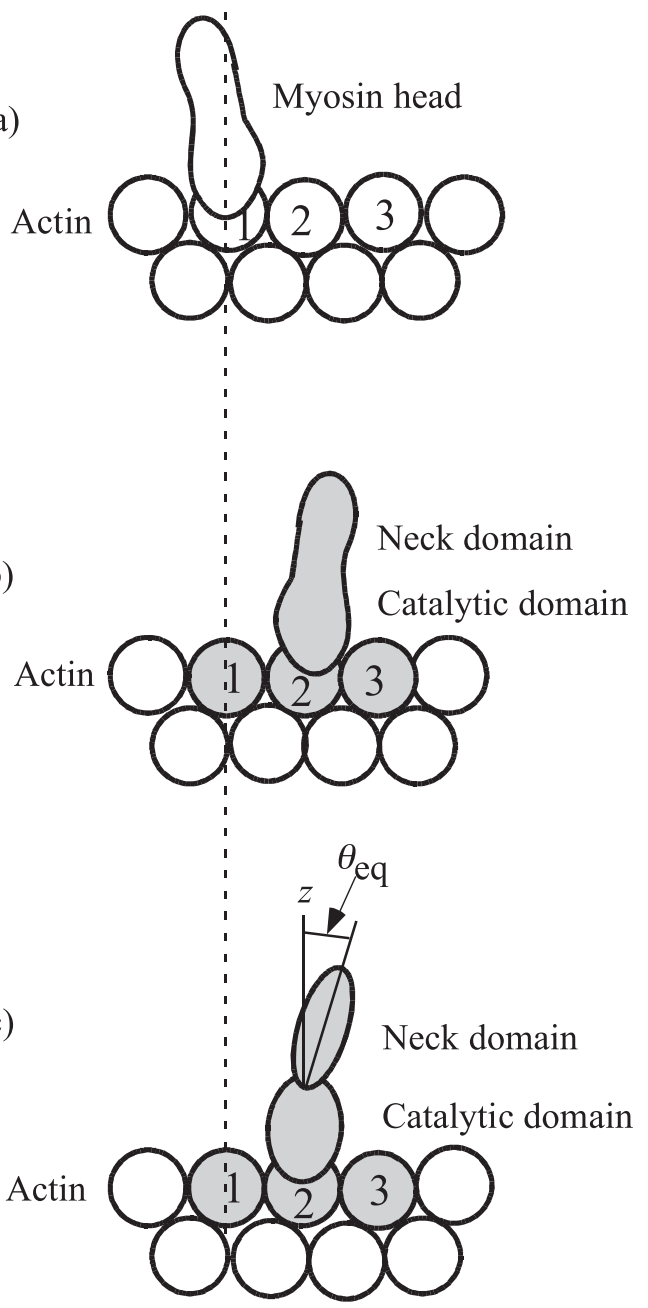

Figure 3 Formation of a complex $\mathrm{MA}_{3}$ of myosin head and actin molecules. (a) Just after attachment of myosin head to actin molecule 1 corresponding to Figure 2(b). Molecules are not deformed yet. (b) The shadowed myosin head and actin molecules 1, 2 and 3 are deformed and the complex $\mathrm{MA}_{3}$ is formed, corresponding to Figure 2(d). (c) Modeling of (b). The myosin head is represented as neck domain plus catalytic domain. The angle $\theta_{\mathrm{eq}}$ is the bending angle of neck domain at equilibrium.

large in the absence of the force on the head but there is some variation of the force depending upon experimental conditions and causes the difference of the number of steps.

Figure 3 illustrates the change of molecular structures associated with the change in potential distribution in Figure 2. Figure 3(a) shows a myosin head just after attachment to actin filament (the shape is speculated), which corresponds to the state in Figure 2(b). Then the potential changes occur from Figure 2(b) to (c) and (d), causing the shift of the binding site of the head from Figure 3(a) to (b). The shape of the head in (b) is depicted referring to the shape in Figure 6 of the paper by Rayment, et al. ${ }^{22}$. As in Figure 2(d), it is assumed that the molecular deformation occurs mainly in the shadowed three actin molecules together with the head. 
The shadowed complex in Fgure 3(b) is denoted as $\mathrm{MA}_{3}$. Figure 3(c) is a modeling of (b). The tilting angle of the neck domain relative to the vertical $z$ axis is denoted as $\theta_{\text {eq }}$ in the case of a single myosin head.

\subsection{Cooperative interaction among crossbridges}

As mentioned in Section 3, the experimental observations indicate that crossbridges produce force independently in isometric tetanus muscle. Based on this observation it is commonly assumed that the myosin head produces force independently in any contracting muscles. This assumption, however, seems too speculative because the experiments were done in the special case of clamped length. Below we shall discuss contracting muscles in general and treat the length-clamped experiment as a specific case that the sliding velocity is zero.

In our model, $41 \%$ of the myosin heads are bound to actin filaments as indicated by Eq. 14. Then crossbridge may appear in one of the three states shown in Figure 4. The complex $\mathrm{MA}_{3}$ is depicted by shadowing and connected to a myosin filament by the thin bar denoted as tail. The end of the crossbridge at the myosin filament is indicated as $\mathrm{K}$ and that at the actin filament as $\mathrm{J}$. The right direction corresponds to the sliding direction of the myosin filament against the actin filament and is called forward and the left direction backward. The tail has bending freedom (cf. Sect. 3.4 of ref. 2), which is represented by one hinge depicted as a black square for simplicity. The force which the crossbridge exerts on the myosin filament is denoted as $p(y)$, where $y$ is shortening of the crossbridge. In Figure 4(a), the tilting angle of the neck domain is equal to the equilibrium angle $\theta_{\mathrm{eq}}$ and the crossbridge produces no force $(p(y)=0)$. In (b), the myosin filament is set in left from (a) and the neck domain tilts to left from $\theta_{\text {eq }}$ and pulls the myosin filament forward $(p(y)>0)$. The tail is straightened and the elastic force by the myosin head will be straightforwardly transmitted to the myosin filament. In (c), the myosin filament is set in right from (a) and the crossbridge pushes the myosin filament backward $(p(y)<0)$. The tail is bent and the effect of the backward force by the crossbridge will be weakly transmitted to the myosin filament.

As shown in Figure 2(d), the myosin molecule has to cross over the potential barrier $U^{*}$ to move from well 2 to 3 in $\mathrm{MA}_{3}$. In ref. 2, the probability $Q$ that the myosin head crosses over $U^{*}$ is discussed based upon Eyring theory of rate process $^{23}$ as was done by Huxley and Simmons ${ }^{24}$. (It should be noted that our model is quite different from the model discussed in ref. 24, although Eyring theory is used during formulation in both models.) Considering that $U^{*}$ is a function of the shortening of the crossbridge $y, Q$ is given by

$$
Q(y)=A \exp \left(-U^{*}(y) / k T\right)
$$

where $A$ is a constant, $k$ the Boltzmann constant and $T$ the absolute temperature. In ref. $2, U^{*}(y)$ is expressed by (a)

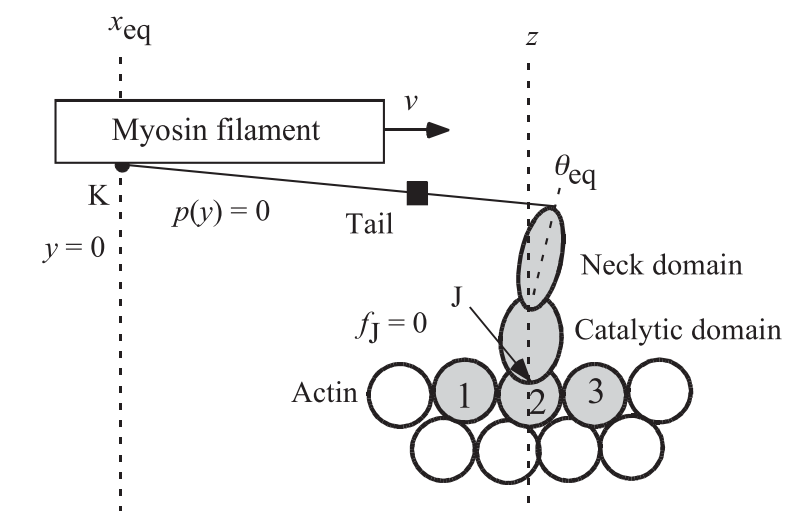

(b)

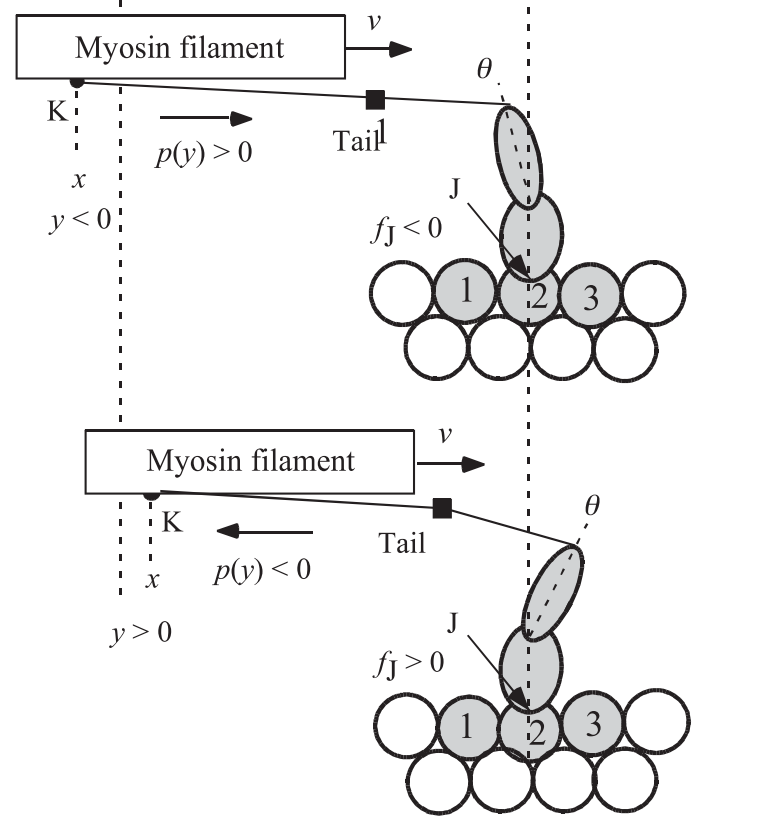

Figure 4 Three states (a), (b) and (c) of the $\mathrm{MA}_{3}$ complex indicated by shadowing. Ends of the crossbridges are indicated as J and $\mathrm{K}$. Ends $\mathrm{J}$ are set on the dotted line $z$ and $\mathrm{K}$ at different positions referring to the dashed line $x_{\text {eq }}$ in (a), (b) and (c). $y$ represents shortening of the crossbridge. (a) The myosin head is at its equilibrium angle, $\theta_{\text {eq }}$ and $y=0$. (b) The myosin head is pulling the myosin filament forward. The crossbridge is elongated and $y<0$. (c) The myosin head is pushing the myosin filament backward. The crossbridge is shortened and $y>0$.

$U^{*}(y)=U^{*}{ }_{0}-a f_{\mathrm{J}}(y)\left(\right.$ Eq. 3-5-2) and $f_{\mathrm{J}}(y)=-p(y)($ Eq. 3-4-2), where $U^{*}{ }_{0}$ and $a$ are constants. Thus we have

$$
U^{*}(y)=U_{0}^{*}+a p(y)
$$

The stiffness of the elongated crossbridge (i.e., myosin head + tail) is denoted as $\kappa_{\mathrm{f}}$ in the case of Figure 4(b) and that of the bent crossbridge in (c) as $\kappa_{\mathrm{b}}$. Then, following Eq. 3-4-6 in ref. $2, p(y)$ is given by

$$
\begin{aligned}
& p(y)=-\kappa_{\mathrm{f}} y, \text { for } y<0 \\
& p(y)=-\kappa_{\mathrm{b}} y, \text { for } y>0
\end{aligned}
$$

Values of the stiffnesses are given by Eqs. 4-1-13 and 4-114 in ref. 2: 
$\kappa_{\mathrm{f}}=2.80 \mathrm{pN} / \mathrm{nm}$
$\kappa_{\mathrm{b}}=0.26 \mathrm{pN} / \mathrm{nm}$

Eq. 19 means that the crossridge is elastically non-linear.

We leave mathematical discussion based upon these equations to ref. 2, and explain only implication of the equations here. Figure 5 illustrates what happens with the model during the filament sliding. Figure 5(a) shows the state that $p(y)<0$ and the stiffness is $\kappa_{\mathrm{b}}$. (The head corresponds to the weakly attached head in term of the power stroke model.) When the myosin filament moves forward, $p(y)$ negatively increases and thus the potential barrier $U^{*}(y)$ decreases by Eq. 17 and the transition probability $Q$ increase by Eq, 16 . As an statistical average, there may be a critical $y$ (denoted as $y_{\mathrm{c}}$ ) at which the head crosses over $U^{*}$. Figure 5(b) shows the state after the head crosses over $U^{*}$ and forms a new complex $\mathrm{MA}_{3}$. Now $p(y)>0$ and the stiffness is $\kappa_{\mathrm{f}}$. (The head corresponds to the strongly attached head in term of the power stroke model.) $U^{*}(y)$ is large by Eq. 17 and $Q$ is small by Eq. 16. Hence the head does not cross over $U^{*}$ and pulls the myosin filament at the same position. The myosin filament continues to move forward and the state (c) is realized. The situation at (c) is identical to (a) except for binding actin mates. Filament sliding takes place repeating such steps. Since the stiffness $\kappa_{\mathrm{f}}$ is much larger than $\kappa_{\mathrm{b}}$ (cf. Eq. 19), the time-averaged force produced by the crossbridge becomes positive.

The filament sliding causes the shift of $\mathrm{MA}_{3}$, while the filament sliding is a result of force production by all $\mathrm{MA}_{3}$. Thus the force production of each crossbridge is helped by other crossbridges. In this sense, there is a mutual cooperation among crossbridges. Generally cooperative system is mathematically discussed by using a feedback loop that output of each element is affected by output of all elements in the system (cf. for instance, Sect. 22 of ref. 25). Eqs. 16 19 imply such a feedback loop.

Cooperative interaction among crossbridges is caused by the filament sliding and disappears when the sliding stops. Accordingly, crossbridges produce force independently in the isometric tetanus state, in agreement with the observation by Ramsey and Street $^{8}$ and Gordon et al. ${ }^{9}$.

\section{4 energy liberation and chemical reactions associated with force production}

Lymn and Taylor ${ }^{26}$ proposed the chemical cycle relating the force production in the power stroke model. In their scheme, dissociations of $\mathrm{Pi}$ and ADP play the important role in force production. In our model, the way of energy flow and chemical reaction is quite different from theirs and dissociations of Pi and ADP do not play any important role in connection with force production. Portions of the ATP hydration energy $e_{\mathrm{A}}$ stored in the head are used in formation the complex $\mathrm{MA}_{3}$ (cf. Fig. 3(b)) and for the force generation steps. Each step of force generation is associated with the chemical reactions: dissociation from one actin molecule (a)

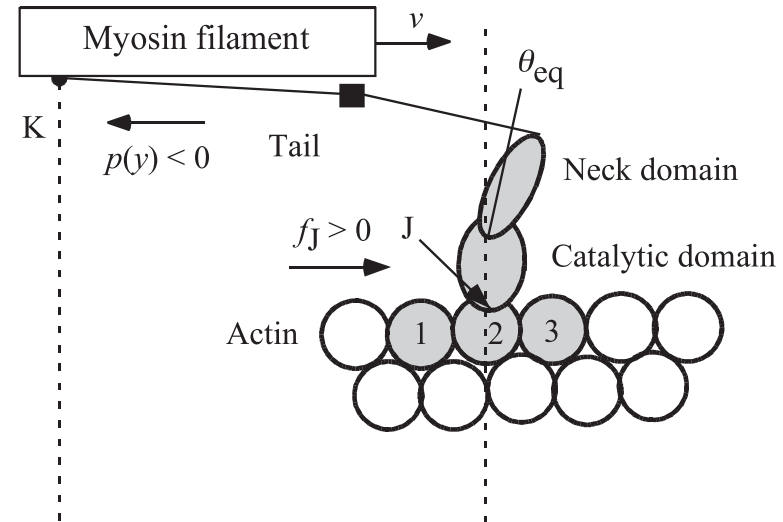

(b)

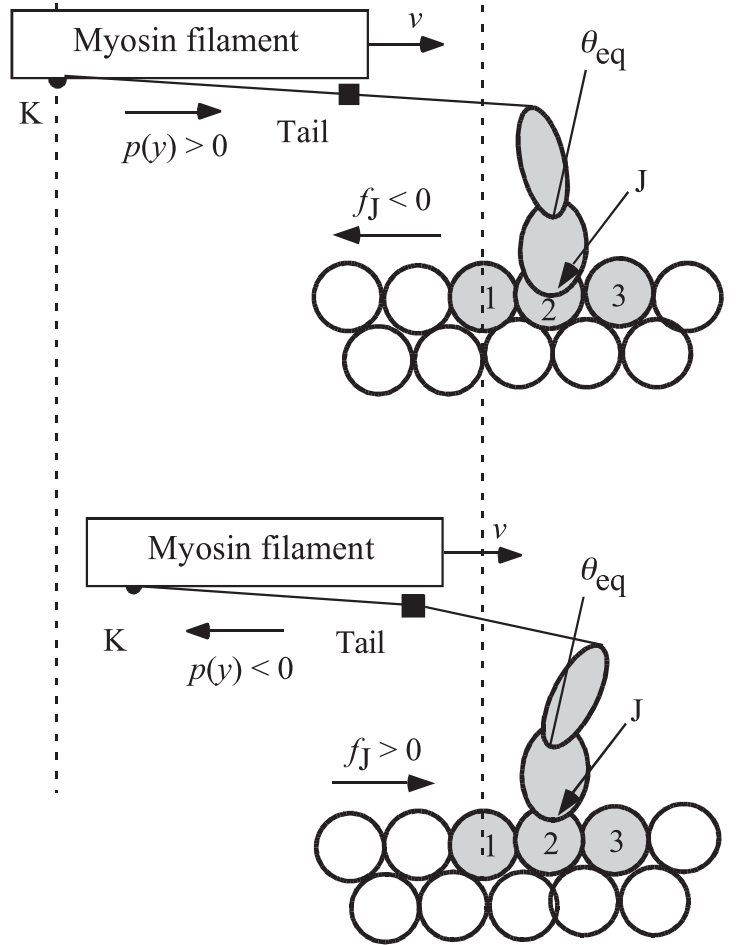

Figure 5 Step motion of complex $\mathrm{MA}_{3}$ (shadowed) along an actin filament. (a) The neck domain tilts to the right and $\mathrm{MA}_{3}$ is just before translation to the right. (b) Just after the $\mathrm{MA}_{3}$ has moved to the new position. The crossbridge is pulling the myosin filament forward. (c) The neck domain tilts to the right and $\mathrm{MA}_{3}$ is ready for next translation to the right.

and binding to the neighboring actin molecule. Figure 6 is an illustration of the steps. On the right, myosin head is depicted as a box with the energy $G$ stored in it. The level of $G$ is lowered successively associated with the step of force production of the myosin head. An actin filament is depicted as a box with many shelves on the left. Shelf $\mathrm{A}_{\mathrm{i}}$ corresponds to $i$-th actin molecule. The force $f_{\mathrm{J}}$ from the myosin filament to the head lowers the potential barrier $U^{*}$ and lets the head dissociate from $A_{i-1}$ and bind to $A_{i}$. With this step, a myosin head produces force by spending the energy indicated as $g$. Calculation result on this scheme is shown later in Figure 9. The step energy liberation seems to be related with thermal 


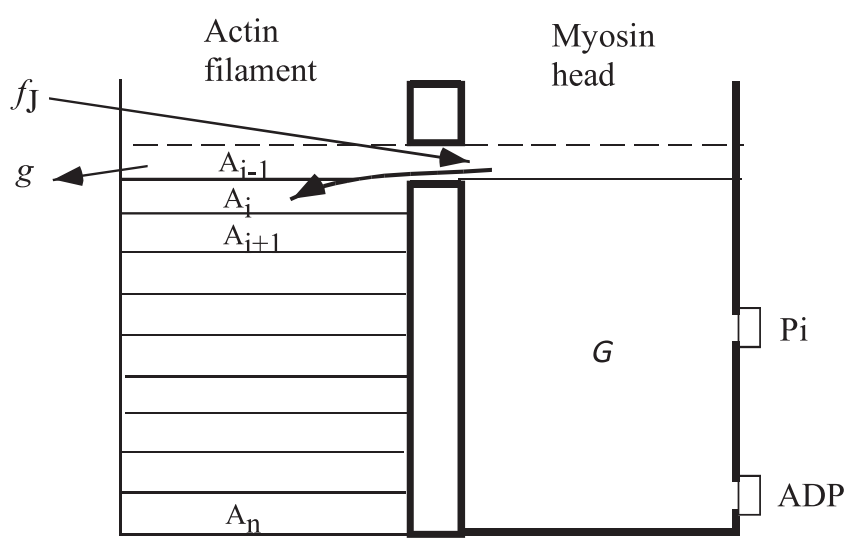

Figure 6 Energy flow and chemical reaction associated with the force production. Chemical reaction is a series of detachment from actin $\mathrm{A}_{\mathrm{i}-1}$ and attachment to $\mathrm{A}_{\mathrm{i}}$. The fraction, $g$, of the free energy of a myosin head is used for force production at each chemical reaction. The energy stored in the myosin head after the i-th step is denoted by $G$. The initial value of $G$ is $e_{\text {A }}$ given to the head by the ATP hydrolysis.

fluctuations as discussed in next section.

\subsection{Role of thermal fluctuations}

Based upon Eyring theory of rate process ${ }^{23}$, the probability $Q$ for a myosin head to cross over $U^{*}$ is given by $Q(y)=A \exp \left(-U^{*}(y) / k T\right)$ (Eq. 16). In usual textbooks, Eyring theory is discussed in case that a single material particle crosses over a potential barrier $U^{*}$. Then, the energy for the particle to cross over $U^{*}$ is supplied by the thermal energy of the surroundings. In the case of a myosin head, however, the head has much internal freedom for structural and thermal fluctuation. Then it becomes possible that the fluctuation occurs adiabatic in a limited time scale in the way that the decrease of structural fluctuation energy is compensated by increase of the thermal fluctuation energy of constituent atoms, and vise versa. Thus the internal energy of the head can be used to excite the mode of motion of center of gravity of the head and to help the head to cross over $U^{*}$ in Figure 2(d). Then the difference between the potential energies at wells 2 and 3 is converted into the elastic energy of the crossbridge to be used to pull the myosin filament. In this way the internal energy of the head originally supplied by ATP hydrolysis is used by parts to pull the myosin filament. The thermal fluctuation plays an important role for force production but it is not against the second law of thermodynamics because contribution from thermal fluctuation of surroundings to force production is zero as time average.

This idea is similar to the model proposed by Huxley in $1957^{27}$ in the sense that the thermal energy plays an important role to produce force. In Huxley model, however, it is assumed that excitation of thermal fluctuation having energy comparable to the ATP hydration energy $e_{\mathrm{A}}$ (about $21 k T$ ) occurs to produce the power stroke. Then the probability for such excitation is approximately proportional to $\exp \left(-e_{\mathrm{A}} / k T\right)=\exp (-21)=7.6 \times 10^{-10}$, which seem too small to be realistic. In our model, a fraction of $\varepsilon_{\text {ATP }}$ is used in each cycle of force production and there is no such difficulty.

\section{Comparison of calculation results with experimental data}

In this section it is shown that calculations based upon our model well explain observed relations between muscle properties or behaviors. The presented figures are reproduction of the figures in refs. 2, 3. Readers may refer to refs. 2, 3 for detailed discussion on the calculations.

The ratio of the stiffness of the muscle at $T=0$ to that at $T=T_{0}$ is about 0.35 where $T_{0}$ is the isometric tension. This $T$ dependency of stiffness was attributed to the variation of the number of the attached myosin heads in the power stroke model. In our model, the $T$-dependency is explained based upon the difference between the stiffness $\kappa_{\mathrm{f}}$ of the crossbridge having the straightened tail and $\kappa_{\mathrm{b}}$ for the bent tail (cf. Eq. 19). Figure 7 shows the calculated relative stiffness $S / S\left(T_{0}\right)$ of muscle as a function of $T / T_{0}$, in comparison with the experimental data.

The force-velocity relation is calculated in Sect. 4.2 of ref. 2 based upon Eqs. 16 19. Figure 8 shows the result by the red curve in comparison with the experimental data by Edman $^{5}$. The data points deviate from the red curve as the tension increases beyond $\mathrm{T}_{\text {dev }} / \mathrm{T}_{0}=0.66$. The origin of this deviation is discussed later with Figures 11 and 12.

The energy liberation rate is discussed in Sect. 4.3 of ref. 2. The obtained expression for the rate is given by Eq. 4-313 in ref. 2. The functional form of the expression is quite similar to the empirical equation for the rate given by $\mathrm{Hill}^{30}$. Figure 9 shows calculation result in ref. 2 in comparison with the experimental data.

Figure 10 shows the distance $D$ over which a myosin

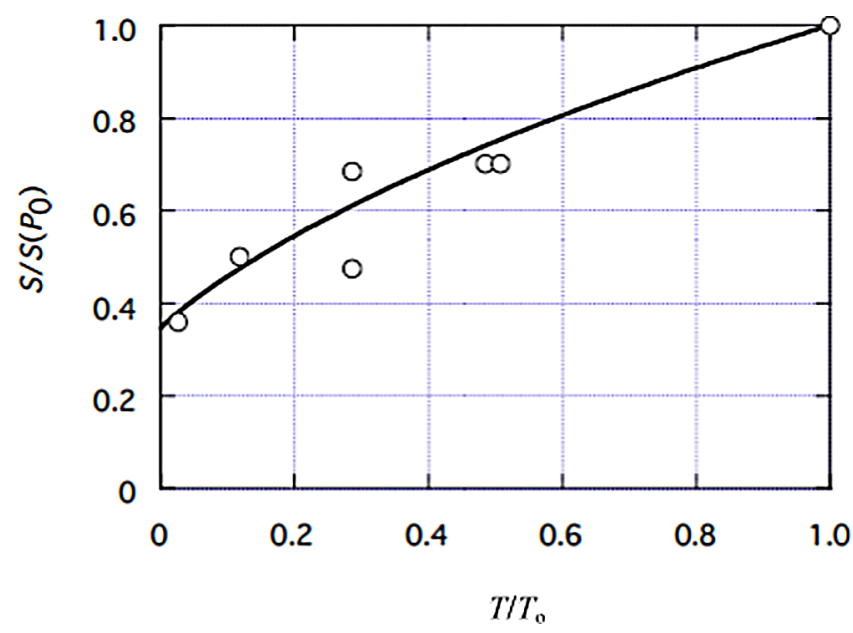

Figure 7 Relative stiffness $S / S\left(T_{0}\right)\left(=S / S\left(P_{0}\right)\right.$ on the ordinate $)$ of muscle as a function of $T / T_{0}$, after Figure 9 of ref. 2. Open circles: Experimental data cited from Figure 10B of the paper by Ford et al. ${ }^{28}$. Solid line: Calculation results in ref. 2. 


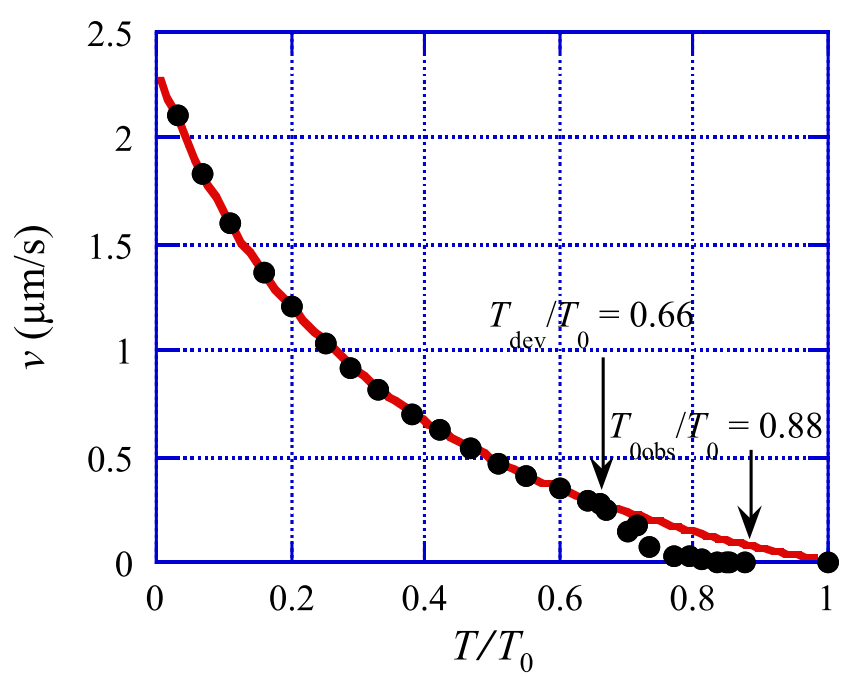

Figure 8 Force-velocity relation and definition of $T_{\text {dev }}$ and $T_{0 \text { obs }}$, after Figure 11 of ref. 2. The ordinate $v$ is the filament sliding velocity per half sarcomere. The red curve shows result of calculation in ref. 2, which coincides with the hyperbolic force-velocity relation reported by Hill ${ }^{29}$. Circles correspond to experimental results given in Figure $6 \mathrm{~A}$ of the paper by Edman ${ }^{5}$.

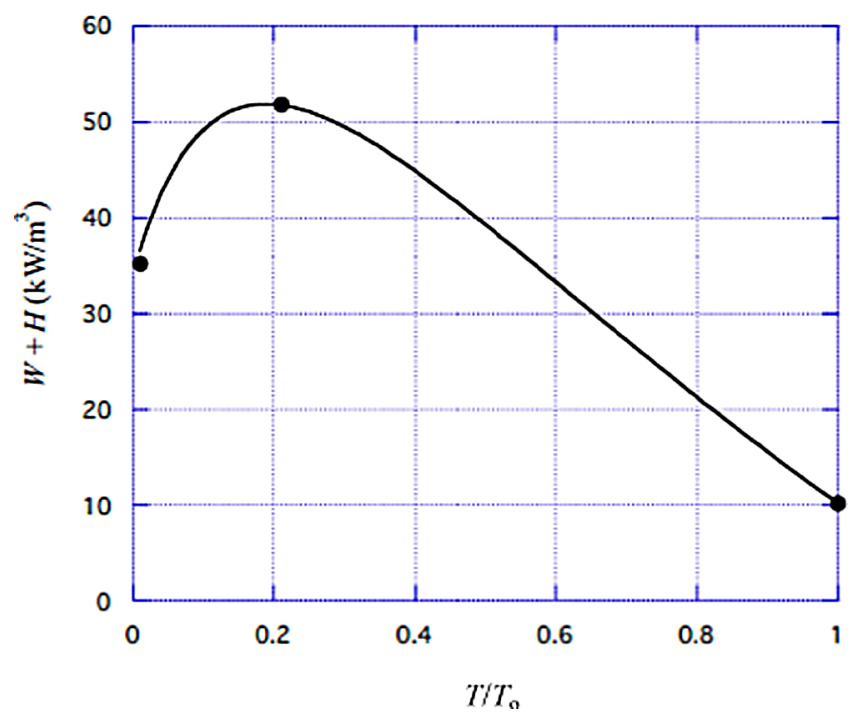

Figure 9 Energy liberation rate $W+H$ in contracting muscle as a function of $T / T_{0}$, after Figure 12 of ref. 2. Black circles: experimental data by Homsher et al. ${ }^{6}$. Curve: results of calculation in ref. 2 .

head translates using $e_{\mathrm{A}}$. Calculation was done ${ }^{2}$ by using Eqs. 2, 4 and 14 and the values given by the curves in Figures 8 and $9 . D$ is $369 \mathrm{~nm}$ at $T / T_{0}=0$.

Large values of $D$ can be obtained also from the experiment by Harada et al. ${ }^{7}$ cited in Sect. 2.4. They observed that most actin filaments shorter than about $40 \mathrm{~nm}$ dissociated from the myosin-coated surface while longer ones remained bound to the surface. This fact suggests that 2 or 3 myosin heads are bound to an actin filament of $40 \mathrm{~nm}$ so that at least one of the myosin heads remains to attach to the filament

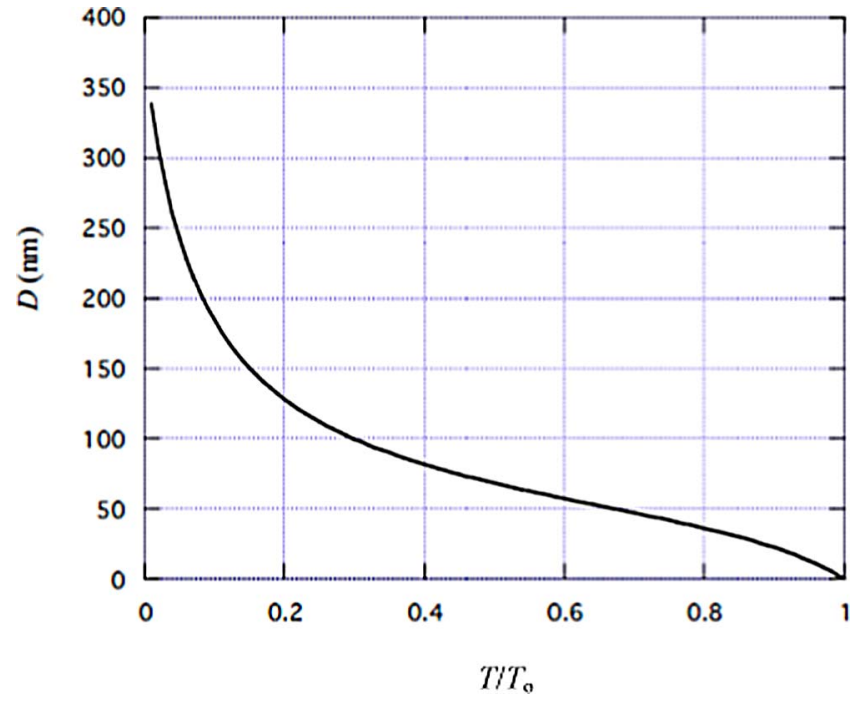

Figure 10 Calculated $D$ as a function of $T / T_{0}$, after Figure 14 of ref. 2. $D$ is the distance over which a myosin head translates using the ATP hydrolysis energy $e_{\mathrm{A}}$. Temperature is about $0^{\circ} \mathrm{C}$.

though the others detach. In Sect. 2.4, the mean distance between neighboring myosin molecules $d_{\mathrm{H}}$ is set equal to $12 \mathrm{~nm}$ (Eq. 8). Then the number of the myosin heads which are under the actin filament of $40 \mathrm{~nm}$ is $2 \times 40 / 12=6.7$ considering two heads of the myosin molecule. Let us denote the ratio of the number of myosin heads attached to the actin filament per that of all myosin heads under the actin filament as $r_{\mathrm{H}}$ as in Eq. 7. Then we have $6.7 r_{\mathrm{H}}=2 \sim 3$. Thus $r_{\mathrm{H}}=0.30 \sim 0.45$. By using values of $D / r_{\mathrm{H}}=850,350,1280 \mathrm{~nm}$ in Eq.11, we have $D=260 \sim 380,110 \sim 160.380 \sim 580 \mathrm{~nm}$ at $T / T_{0}=0$. They are as large as $369 \mathrm{~nm}$ at $T / T_{0}=0$ in Figure 10. Also Harada et al. ${ }^{7}$ observed that an actin filament of about $40 \mathrm{~nm}$ sometimes stopped the movement. Presumably this is the case that only one myosin head remains to attach to an actin filament so that there is no cooperatively working mate and the actin filament stops.

Figure 2(d) schematically shows the general case that the existence probability of the myosin head is distributed in wells 1 and 2. Figure 8 shows that the experimental data deviate from the calculation result (the red curve) for $T / T_{0}>$ $T_{\text {dev }} / T_{0}=0.66$. In ref. 3 , the origin of this deviation is attributed to variation of the existence probability of the myosin head in $\mathrm{MA}_{3}$ complex. Figure 11 illustrates the two cases of the probability distribution. In the case (a), the myosin head exists solely in well 2 . This case is discussed in ref. 2 and leads us to the red curve in Figure 8 as well as the curves in Figures 7, 9 and 10. In the case (b) the existence probability of the myosin head is finite in well 1 . This case is discussed in ref. 3. Eqs. 17 19 mean that $U^{*}$ decreases and stimulates forward motion of the myosin head when forward force on the head increases. Similarly, $U^{*}{ }_{12}$ decrease when backward force on the head increases as $T$ approaches $T_{0}$ in Figure 11b. Here it should be noted that implication of the potential 
(a)

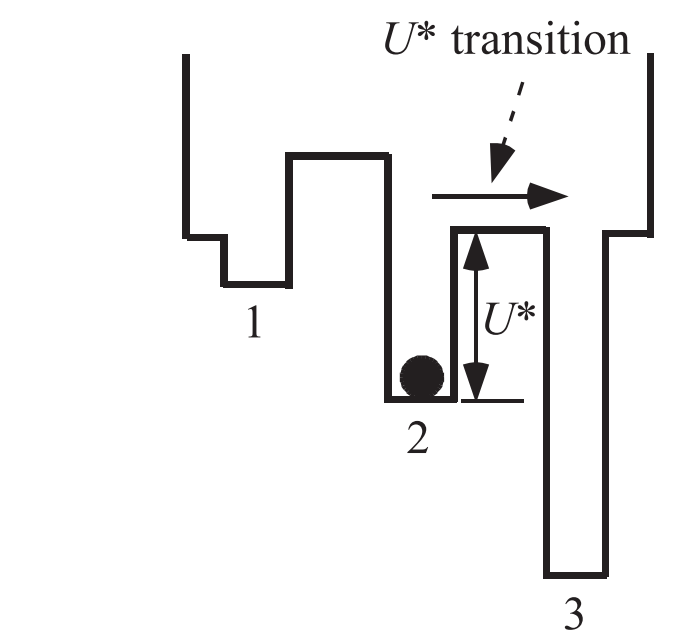

(b)

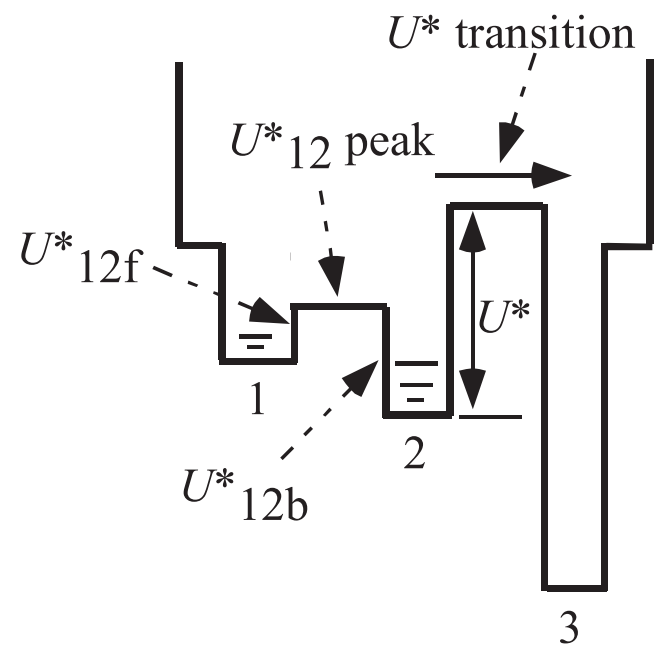

Figure 11 The potential of force exerted on the myosin head in $\mathrm{MA}_{3}$ complex. The potential shape varies depending upon the tension $T$. (a) The case of $T<T_{\text {dev }}$. The black circle indicates that the myosin head exists solely in well 2 . The force-velocity relation is Hill-type in this case. (b) The case of $T_{\mathrm{dev}}<T<T_{\text {oobs}}$, where the existence probability of the myosin head is distributed in wells $\mathrm{A} 1$ and $\mathrm{A} 2$, causing deviation of the force-velocity relation from Hill-type.

distribution in Figure 11 is quite different from the potential diagram in Figure 6 of the paper by Huxley and Simmons ${ }^{24}$ although they are somewhat similar in appearance.

In Sect. 4.3, the potential barrier $U^{*}$ is expressed by $U^{*}(y)=U^{*}+a p(y)$ (Eq. 17). This expression is used in combination with the assumption that the myosin head exists solely in well 2 as shown in Figure 11(a). In Sect.7 of ref. 3, it is discussed how the potential height $U^{*}(y)$ varies when the existence probability of the myosin head becomes finite in well 1 as shown in Figure 11(b). As a result, $U^{*}(y)$ is given by

$$
\left.U^{*}(y)=U^{*}{ }_{0}+a p(y)+c\left(y_{\mathrm{c}}{ }^{\mathrm{H}}-y_{\mathrm{c}}{ }^{*}\right)\right)
$$

Here $c$ is a constant and $\left(y_{\mathrm{c}}{ }^{\mathrm{H}}-y_{\mathrm{c}}{ }^{*}\right)$ is a parameter concerning (a)

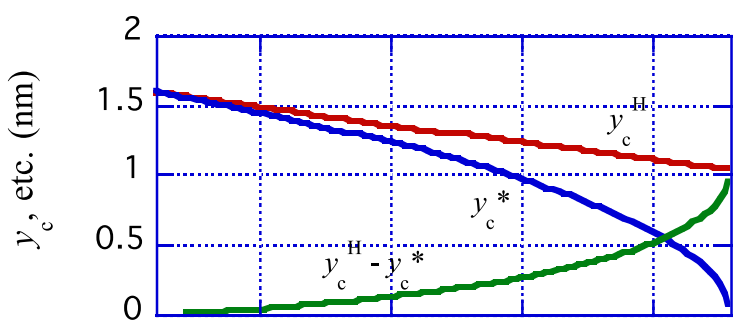

(b)

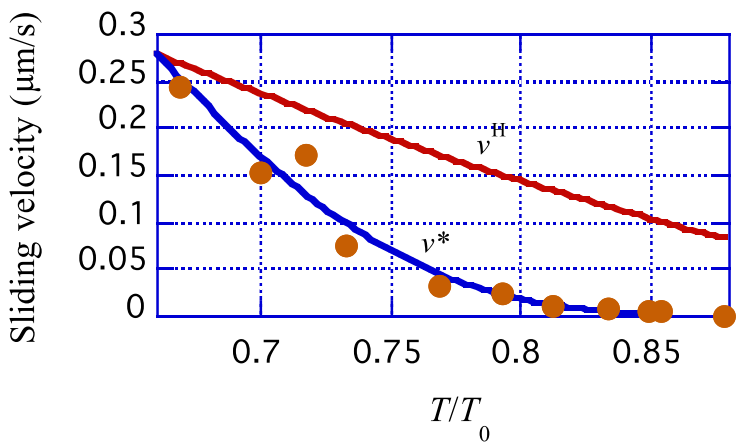

Figure 12 Deviation of the force-velocity relation from the Hilltype, after Figure 19 of ref. 3. (a) The green curve gives $y_{\mathrm{c}}{ }^{\mathrm{H}}-y_{\mathrm{c}}{ }^{*}$ which appears in Eq. 21. (b) Brown circles: Experimental data of the sliding velocity per half sarcomere from Figure $3 \mathrm{~A}$ of the paper by Edman ${ }^{5}$. Red curve: $v^{\mathrm{H}}$, the Hill-type filament sliding velocity, the same as the red curve in Figure 8. Blue curve: $v^{*}$, the velocity calculated by Eqs. 21 and 22.

structural modulation of $\mathrm{MA}_{3}$ due to finite existence probability of the myosin head in well 1 . Calculated $\left(y_{\mathrm{c}}^{\mathrm{H}}-y_{\mathrm{c}}{ }^{*}\right)$ is given as a function of $T / T_{0}$ by the green curve in Figure 12(a). The filament sliding velocity $v^{*}$ to be observed is given in relation with the Hill-type velocity $v^{\mathrm{H}}$ by

$$
v=v^{\mathrm{H}} \exp \left(-b_{v}\left(y_{\mathrm{c}}{ }^{\mathrm{H}}-y_{\mathrm{c}}{ }^{*}\right)\right)
$$

Calculation is done by setting

$$
b_{v}=c / k T=7.9(1 / \mathrm{nm})
$$

Obtained $v$ is shown by the blue curve Figure $2 \mathrm{~b}$ in comparison with the Experimental data by Edman ${ }^{15}$.

Various workers studied the isometric tension transient and isotonic velocity transient. In ref. 3, calculations are done to explain the experimental data on isometric tension transient by Ford et al. ${ }^{31}$ and on the isotonic velocity transient by Civan and Podolsky ${ }^{32}$.

Huxley $^{33}$ divided the transient responses to the sudden reduction of length or of load into four phases. The first phase of the isometric tension transient is the step change of tension due to sudden length change. The first phase of the isotonic velocity transient is the step change of muscle length due to the sudden load change. The initial state in these changes is the isometric tetanus state, in which the existence probability of the myosin head is finite in well 1 as shown in Figure 11(b). Calculation is done assuming that the existence probability does not change during the first phases since the length or load changes are very fast. 


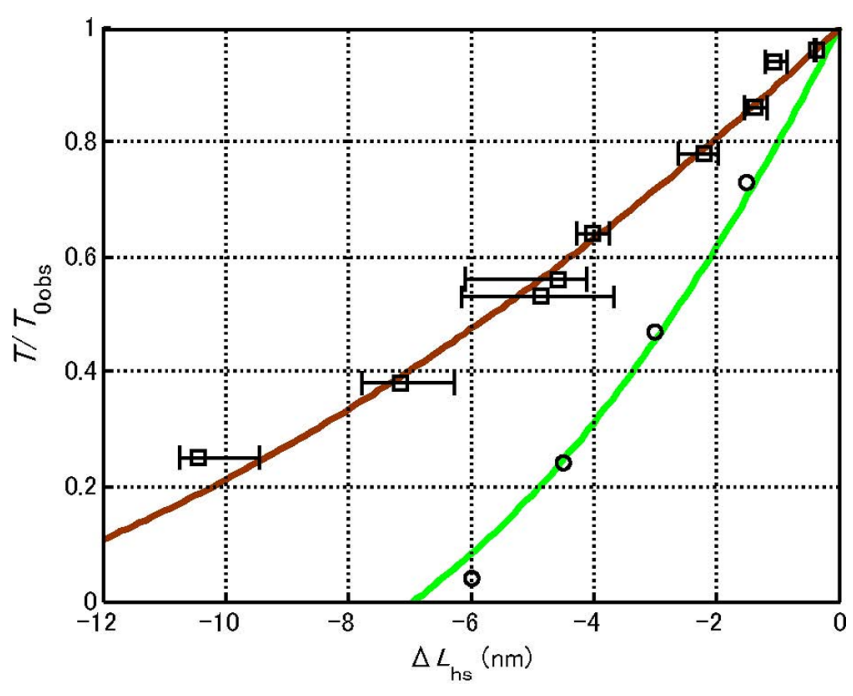

Figure $13 T / T_{\text {0obs }}$ vs. $\Delta L_{\mathrm{hs}}$ relations in the first phases in the transient phenomena, after Figure 9 of ref. 3. Circles: experimental data in the isometric tension transient cited from Figure 13 of Ford et al. ${ }^{31}$ Squares: experimental data in the isotonic velocity transient from Figure 3 of Civan and Podolsky ${ }^{32}$, in which the error bar means error of read-out from Figure 3 of ref. 32. The green curve: $T / T_{\text {oobs }}$ calculated for the first phase of the isometric tension transient in ref. 3 . The brown curve: $\Delta L_{\mathrm{hs}}$ calculated for the first phase of the isotonic velocity transient in ref. 3 .

Figure 13 shows calculation results reported in Sect. 4 of ref. 3 . Here the length change per half sarcomere is denoted as $\Delta L_{\mathrm{hs}}$ and the relative tension is given by $T / T_{0 \text { obs }}$ in both transients (cf. Figure 8 for $T_{0 \text { obs }}$ ). The experimental data by Ford et $a^{31}$ are shown by circles for the isometric tension transient and the data by Civan and Podolsky ${ }^{32}$ by squares for the isotonic velocity transient. Calculation results are shown by the green curve for the isometric tension transient and by the brown curve for the isotonic velocity transient. Calculation is done by using the values of stiffnesses given by Eq. 19, which implies that the elasticity of crossbridge is largely nonlinear. The curvatures of the curves in Figure 13 reflect the nonlinearity. To get good agreement with the experimental data, it is assumed that extensibility ratios of the crossbridge, myosin filament and actin filament are $0.22,0.26$ and 0.52 , respectively. A comment is given on these values in Sect. 6 .

As mentioned above, the curves in Figure 13 are obtained assuming that the existence probability of the myosin head in well 1 of Figure 11(b) does not change during the first phases. Thus the structure of $\mathrm{MA}_{3}$ becomes unstable just after the first phases. The complex muscle behaviors after the first phases are considered to correspond to the process that the existence probability of the myosin head in $\mathrm{MA}_{3}$ changes into the stable probability at a given condition.

Figure 14 shows $T / T_{\text {0obs }}$ as functions of time $t$ for various length change steps $\Delta L_{\mathrm{hs}}$ after the first phase in the isometric tension transient. Sect. 5 of ref. 3 gives mathematical details on the calculation.
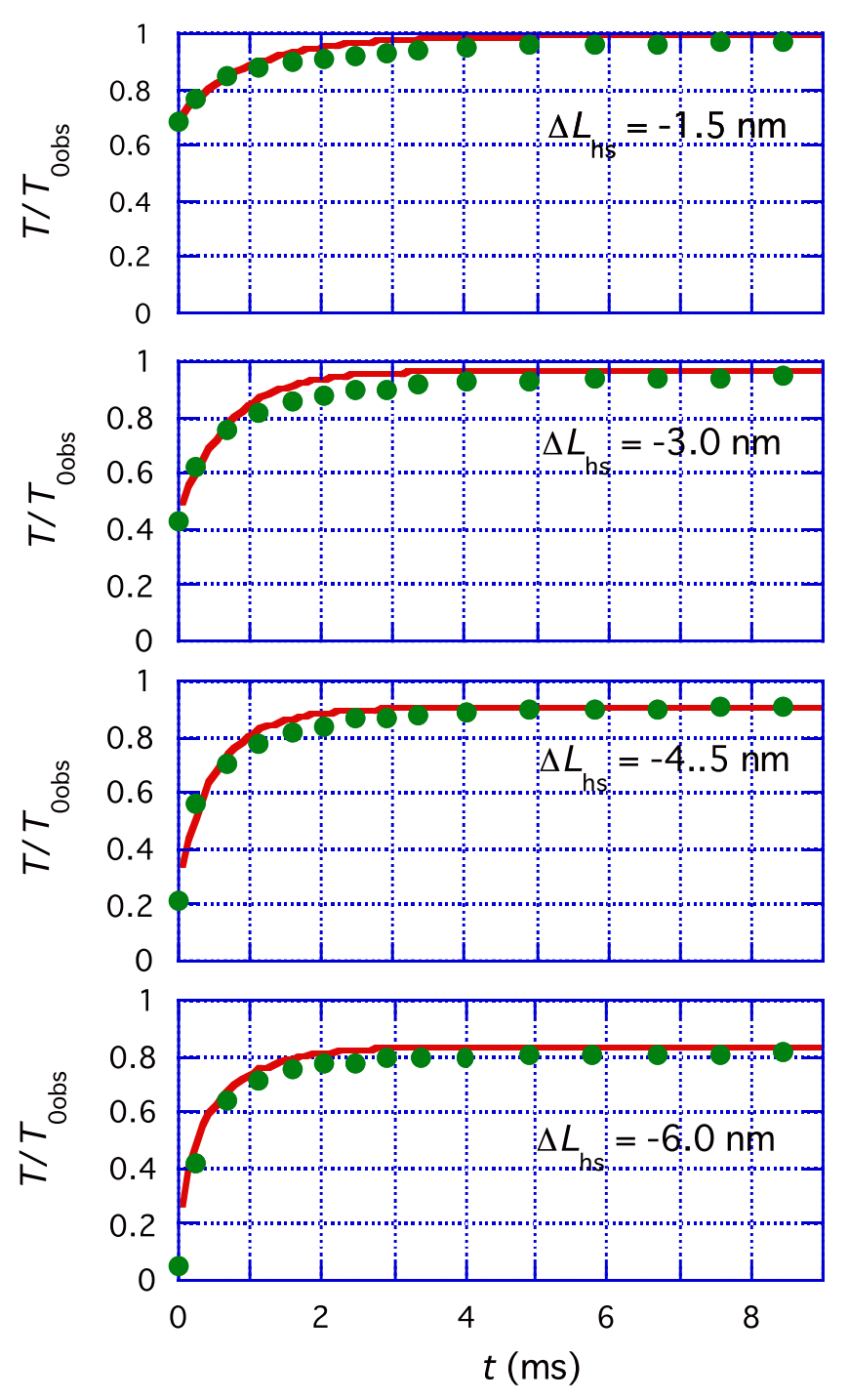

Figure $14 T / T_{\text {obs }}$ as functions of time $t$ for various length change steps $\Delta L_{\mathrm{hs}}$ in the isometric tension transient, after Figure 14 of ref. 3. The origin of $t$ is set as the moment that the first phase finishes. Green circles: Experimental data cited from Figure 23 of Ford et al. ${ }^{31}$. Red lines: Calculation results in ref. 3.

Figure 15 shows time course of length change $\Delta L_{\mathrm{hs}}$ ' after the first phase in the isotonic velocity change. Sect. 6 of ref. 3 gives mathematical details on the calculation.

\section{Summary and Discussion}

In Sect. 3, requirements for any models based upon the filament sliding theory are listed. They are discussed with the same item numbers.

(1) Large $D / r$ in shortening muscle

Note that the large magnitude of $D / r$ such as $900 \mathrm{~nm}$ (Eq. 6 ) is obtained based upon the sliding filament theory itself and thus any model based upon the sliding filament theory should give such large $D / r$. Large values of $D / r$ are obtained also from the experimental data by Harada et $a .^{7}$ in Sect. 
(a)

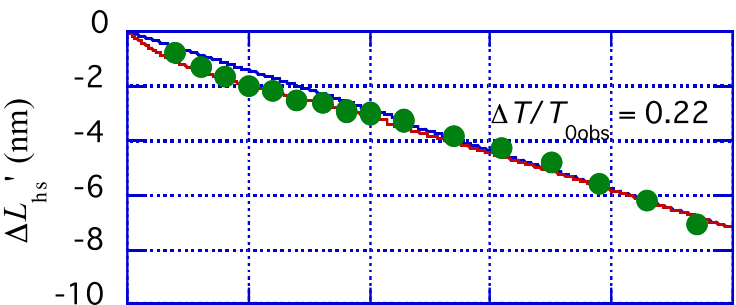

(b)

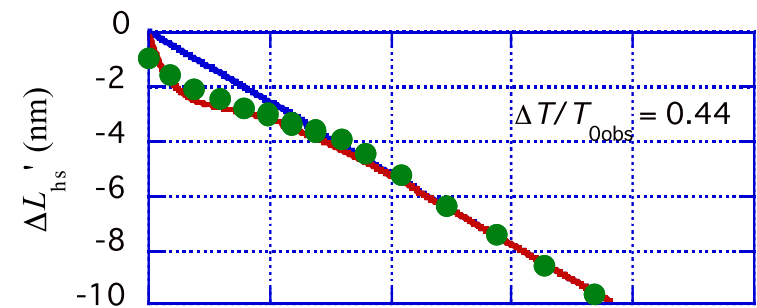

(c)

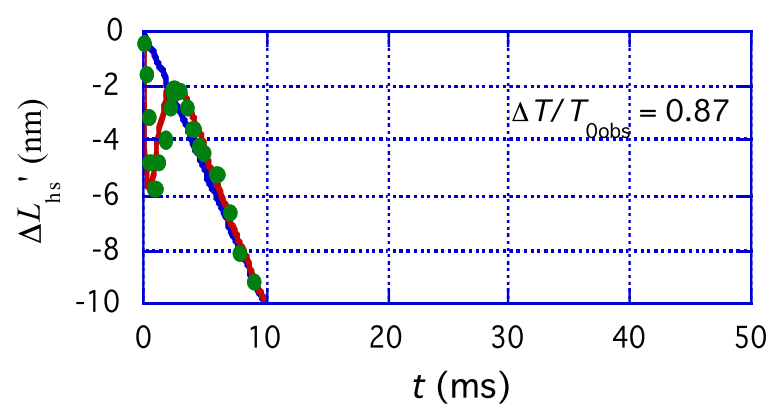

Figure 15 Length change $\Delta L_{\mathrm{hs}}$, vs. $t$ for three values of $\Delta T / T_{0 \mathrm{obs}}=$ $\left(T_{0 \text { obs }}-T\right) / T_{\text {oobs }}$, after Figure 18 of ref. 3. (a) $\Delta T / T_{0 \text { obs }}=0.22$. (b) $\Delta T / T_{0 \text { obs }}$ $=0.44$. (c) $\Delta T / T_{\text {0obs }}=0.87$. Green data points are obtained from Figure 3 of the paper by Civan and Podolsky ${ }^{32}$. Blue straight lines are for the steady filament sliding ${ }^{32}$. Red curves are the calculation results in ref. 3 .

2.4. Our model is constructed to give the large values of $D / r$.

The order of magnitude of $D$ is about $10 \mathrm{~nm}$ in the power stroke model. Combining $D / r=900 \mathrm{~nm}$ and $D=10 \mathrm{~nm}$ gives $r=0.011$, which seems too small to be realistic. Hence the power stroke model does not seem to fulfill this requirement. More detailed discussion on difficulty of the power stroke model is given in Sect. 2.2 of ref. 2 .

The cooperation between the myosin and actin molecules is important for force production in our model. In the power stroke model, the actin filament is treated as a relatively passive element like a ladder for a myosin head. In general protein molecules seem to play more active role in biological systems.

(2) Chemical reactions associated with the large $D / r$

In our model, the ATP hydration energy is spent by fraction through repeated chemical reactions between the myosin head and actin filament, as illustrated in Figure 6. The small amount of energy liberated per one step make the contribution of the thermal fluctuation possible as discussed in Sect. 4.5. This scheme well explains the energy liberation rate vs. $T / T_{0}$ relation as shown in Figure 9.

(3) Crossbridges produces force independently in the isometric tetanus state
As discussed at the end of Sect. 4.3, the cooperative interaction among crossbridges is caused by the filament sliding and disappears when the sliding velocity becomes zero. Accordingly, crossbridges produce force independently in the isometric tetanus state, in agreement with the observation by Ramsey and Street $^{8}$ and Gordon et al. ${ }^{9}$.

(4) Small $D$ observed in experiments with single myosin molecule in vitro

In the expression of $U^{*}(y)=U^{*}{ }_{0}+a p(y)$ (Eq. 17), $U^{*}{ }_{0}$ is relatively large and the probability for the myosin head to cross over $U^{*}$ is small when the force $p(y)$ on the head is small. As discussed in Sect. 4.2, in the in vitro experiments by Molloy et al..$^{11}$ and Kitamura et al. ${ }^{12}$, the single myosin molecule has no partner of cooperation and the force on the head is close to 0 though there may be some fluctuation of the force depending upon the experimental conditions. Hence $D$ is expected to be about $5.46 \mathrm{~nm}$ or a few times of it.

(5) The model should explain many observed relations between muscle properties or behaviors

Figures 7 9 and 12 15 show good agreement between calculation results based upon the model and the experimental data.

Combining Eq.3 and Eq. 14 gives

$$
p / p_{0}=T / T_{0}
$$

This relation means that we can get an expression of a quantity as a function of macroscopic parameter $T / T_{0}$ when we get an expression of the quantity as a function of microscopic parameter $p / p_{0}$. This was very convenient in theoretical treatment in refs. 2,3 .

To get good agreement between the calculation results and experimental data in Figure 13, it is assumed that extensibility ratios of the crossbridge, myosin filament and actin filament are $0.22,0.26$ and 0.52 . These values are approximately in agreement with the extensibility ratios $0.31,0.27$ and 0.42 estimated through X-ray diffraction by Wakabayashi et al. ${ }^{34}$. The ratio is assumed to be 1,0 and 0 in the power stroke model (cf. ref. 35).

In Figure 2, piezoelectric effect is considered. Protein molecules are structurally polar and any biological system should be treated as a system of four variables (stress, strain, electric field and polarization) from the physical viewpoint, as in the discussion on the flagellar rotary motor in ref. 36.

Merit of the two-headed structure of myosin molecule is discussed in Sect. 5.2 of ref. 2.

Cooperativity among myosin molecules is discussed concerning cytoplasmic streaming in Characean algae in Sect. 5.3 of ref. 2 .

Our model has some similarity with the ratchet model (cf. e.g., ref. 37). In the ratchet model, however, concrete picture seems lacking on chemical reaction and energy liberation process during the force production as asked by Requirement 2 in Sect. 3. Chemical reaction and energy liberation process in our model are illustrated in figure 6 . 
Eqs. 18 and 19 mean elastic nonlinearity of the crossbridge. Recently Kaya and Higuchi ${ }^{38}$ studied nonlinear elasticity of single myosin molecules in myofilament.

\section{Acknowledgement}

The authors are grateful to Prof. H. Yoshimura of Meiji University for his kind help during the course of our work.

\section{References}

1. Mitsui, T. Induced potential model of muscular contraction mechanism and myosin molecular structure. in Adv. Biophys. (Ebashi, S. ed.) vol.36, pp. 107-158 (Japan Sci. Soc. press Elsevier Sci. Pub., Tokyo and Limerick, 1999).

2. Mitsui, T. \& Ohshima, H. Remarks on muscle contraction mechanism. Int. J. Mol. Sci. 9, 872-904 (2008).

3. Mitsui, T. \& Ohshima, H. Remarks on muscle contraction mechanism II. Isometric tension transient and isotonic velocity transient. Int. J. Mol. Sci. 12, 1697-1726 (2011)

4. Mitsui, T. \& Ohshima, H. A self-induced translation model of myosin head motion in contracting muscle I. Force-velocity relation and energy liberation. J. Muscle Res. Cell Motil. 9, 248-260 (1988).

5. Edman, K. A. P. Double-hyperbolic force-velocity relation in frog muscle fibres. J. Physiol. 404, 301-321 (1988).

6. Homsher, E, Irving, M. \& Yamada, T. The effect of shortening on energy liberation and high energy phosphate hydrolysis in frog skeletal muscle. in Contractile Mechanism in Muscle (Pollack, G. H. \& Sugi, H. eds) pp. 865-876 (Plenum, New York, 1984).

7. Harada, Y., Sakurada, K., Aoki, T., Thomas, D. D. \& Yanagida, T. Mechanochemical coupling in actomyosin energy transduction studies by in vitro movement assay. J. Mol. Biol. 216, 49-68 (1990).

8. Ramsey, R. W. \& Street, S.F. The isometric length-tension diagram of isolated skeletal muscle fibers of the frog. J. Cell. Comp. Physiol. 15, 11-34 (1940).

9. Gordon, A. M., Huxley, A. F. \& Julian, F. J. The variation in isometric tension with sarcomere length in vertebrate muscle fibres. J. Physiol. 184, 170-192 (1966).

10. Huxley, A.F. Mechanics and models of the myosin motor. Philos. Trans. R. Soc. Lond. B355, 433-440 (2000).

11. Molloy, J.E., Burns, J.E., Kendrick-Jones, J., Tregear, R. T. \& White, D. C. S. Movement and force produced by a single myosin head. Nature 378, 209-212 (1995).

12. Kitamura, K., Tokunaga, M., Hikikoshi-Iwane, A. \& Yanagida, T. A single myosin head moves along an actin filament with regular steps of 5.3 nanometres. Nature 397, 129-134 (1999).

13. Ishijima, A., Harada, Y., Kojima, H., Funatsu, T., Higuchi, H. \& Yanagida, T. Single-molecule analysis of the actomyosin motor using nano-manipulation. Biochem. Biophys. Res. Comm. 199, 1057-1063 (1994).

14. Matsubara, I., Yagi, N. \& Hashizume, H. Use of an X-ray television for diffraction of the frog striated muscle. Nature 255, 728-729 (1975).

15. Yagi, N., Takemori, S. \& Watanabe, M. An X-ray diffraction study of frog skeletal muscle during shortening near the maximum velocity. J. Mol. Biol. 231, 668-677 (1993).

16. Podolsky, R. J., Onge, S. St., Yu, L. \& Lymn, R. W. X-ray diffraction of actively shortening muscle. Proc. Natl. Acad. Sci. USA 73, 813-817 (1976).
17. Huxley, H.E. Time resolved X-ray diffraction studies in muscle. in Cross-bridge Mechanism in Muscle Contraction (Sugi, H. \& Pollack, G. H. eds.) pp. 391-405 (University of Tokyo Press, Tokyo, 1979).

18. Huxley, H.E. \& Kress, M. Crossbridge behaviour during muscle contraction. J. Musc. Res. Cell Motil. 6, 153-161 (1985).

19. Andreeva, A. L., Andreev, O. A. \& Borejdo, J. Structure of the 265-kilodalton complex formed upon EDC cross-linking of subfragment 1 to F-actin. Biochemistry 32, 13956-13960 (1993).

20. Xiao, M., Andreev, O. A. \& Borejdo, J. Rigor cross-bridges bind to two actin monomers in thin filaments of rabbit psoas muscle. J. Mol. Biol. 248, 294-307 (1995).

21. Oosawa, F. \& Asakura, S. Thermodynamics of the Polymerization of Proteins. pp. 66-67 (Academic Press, New York, 1975).

22. Rayment, I., Holden, H.M., Whittaker, M., Yohn, C.B., Lorenz, M., Holmes, K. C. \& Milligan, R. A. Structure of the actin-myosin Complex and its implications for muscle contraction. Science 261, 58-65 (1993).

23. Eyring, H. Viscosity, plasticity, and diffusion as examples of absolute reaction rate. J. Chem. Phys. 4, 283-291 (1936). [See also Sect. 27.4 The Eyring equations. In: Atkins, P. W. Physical Chemistry, $6^{\text {th }}$ Edition (W. H. Freeman \& Co. and Sumanas, Inc., New York, 1998)].

24. Huxley, A.F. \& Simmons, R. M. Proposed mechanism of force generation in striated muscle. Nature 233, 533-538 (1971).

25. Mitsui, T., Tatsuzaki, I. \& Nakamura, E. An Introduction to the physics of ferroelectrics (Gordon \& Breach, New York, 1976).

26. Lymn, R. W. \& Taylor, E. W. Mechanism of adenosin triphosphate hydrolysis by actomyosin. Biochemistry 10, 4617-4624 (1971).

27. Huxley, A.F. Muscle structure and theories of contraction. Progr. Biophys. Biophys. Chem. 7, 255-318 (1957).

28 Ford, L. E., Huxley, A. F. \& Simmons R. M. Tension transients during steady shortening of frog muscle fibres. J. Physiol. 361, 131-150 (1985).

29. Hill, A. V. The heat of shortening and the dynamic constants of muscle. Proc. Roy. Soc. B126, 136-195 (1938).

30. Hill, A. V. The effect of load on the heat of shortening muscle. Proc. Roy. Soc. B159, 297-318 (1964).

31. Ford, L.E., Huxley, A.F. \& Simmons, R. M. Tension responses to sudden length change in stimulated frog muscle fibres near slack length J. Physiol. 269, 441-515 (1977).

32. Civan, M. M. \& Podolsky, R. J. Contraction kinetics of striated muscle fibres following quick changes in load. J. Physiol. 184, 511-534 (1966).

33. Huxley, A. F. Muscular contraction. J. Physiol. 243, 1-43 (1974).

34. Wakabayashi, K., Sugimoto, Y., Tanaka, H., Ueno, Y., Takazawa, Y. \& Amemiya, Y. X-ray diffraction evidence for the extensibility of actin and myosin filaments during muscle contraction. Biophys. J. 67, 2422-2435 (1994).

35. Irving, M. Give in the filament. Nature 374, 14-15 (1995).

36. Mitsui, T. \& Ohshima, H. Shear stress transmission model for the flagellar rotary motor. Int. J. Mol. Sci. 9, 1595-1620 (2008).

37. Juelicher, F., Ajdari, A. \& Prost, J. Modeling molecular motors. Rev. Mod. Phys. 69, 1269-1281 (1997).

38. Kaya, M. \& Higuchi, H. Nonlinear elasticity and an 8-nm working stroke of single myosin molecules in myofilaments. Science 329, 686-689 (2010). 